\title{
A Weighted Logarithmic Model based Enhancement of Weather Degraded Images
}

\author{
Hitendra Gupta ${ }^{1 *}$, K. K. Sharma ${ }^{2}$ and S. D. Joshi ${ }^{3}$ \\ ${ }^{1}$ JECRC Jaipur, ${ }^{2}$ MNIT Jaipur, ${ }^{3}$ IIT Delhi \\ hitendra_gupta@rediffmail.com,kksharma_mrec@yahoo.com,sdjoshi@ee.iitd.ac.in
}

\begin{abstract}
The lack of information content in the images captured under bad weather dominates the reason of poor visibility more, than due to the overall contrast or other. This paper presents a novel method for enhancement of weather degraded images. The proposed enhancement method is based upon the logarithmic image processing (LIP) framework and human visual system (HVS). The proposed method uses multiple images of the same scene in order to increase the information content and produce a visibly enhanced resultant image. A region based contrast measure called the logarithmic region contrast measure (LRCM) for finding the optimal value of location based parameters of the proposed method and its comparison with other traditional contrast measures has been presented here in this paper. The experimental results on two data sets of weather degraded images are included here in this correspondence.
\end{abstract}

Keywords: Logarithmic image processing (LIP), Image enhancement, contrast measure, human visual system

\section{Introduction}

The information content of the images captured in misty and scattering media such as fog, haze, and smoke are low and hence suffers from degradation in visibility. In such media, the radiance and transmittance from a scene point radically gets diffused and modulated. Enhancement of such images formed by transmitted light [1] or the human visual system (HVS) [2], may not comply with the classical linear image processing (CLIP) techniques [3, $4,5]$. These images acquired under murky atmosphere results in the information content loss which increases with increase in distance [6]. Retrieving or improving the visual information in poor visibility is significant for various machine vision and user-assisted applications such as aircraft traffic, mapping, surveillance, navigation, and on-road visibility. There is, thus, a growing interest in the analysis and enhancing the visibility of weather degraded images [6, 7, $8,9]$.

Several methods have been proposed to restore good visibility. Nayar et al., [7] illustrates that the bad weather effects depends more strongly on the depth of the scenes than the overall contrast. Thus, the traditional enhancement techniques e.g. histogram equalization does not befit here. They conferred on various types of weather conditions and their formation processes and summarized two models of atmospheric scattering - attenuation and airlightthat are most pertinent to a HVS.

*Corresponding author: hitendra_gupta@rediffmail.com 
Using these models they recovered the depth map of the scenes for enhancing the scene. Satherley et al., [10] proposed a method for reversing contrast loss in diffuse daylight illumination conditions based on the principle of scattering that required a priori information about the scene geometry. Kopieka and Yitzhaky [11] restore image contrast for a single image that required a priori knowledge of the blur function. Petterson et al., [12] proposed a method for a single image using linear combination of the direct transmission and the airlight.

Models based on the human visual system (HVS) have also been used for image enhancement [13]. Some of these methods attempts to discriminate the edge and non-edge pixels by extracting the edge information of an image [14, 15]. A few HVS based image enhancement model segments an image based upon background intensity and gradient [16], [17].

Thus, due to the deficiency of a broad-spectrum hypothesis of image enhancement, the enhancement task of images is complicated. A successful automatic image enhancement requires an objective criterion for enhancement and an external evaluation of quality [18], [19].

In this paper, we propose a novel method to image enhancement of weather degraded images on the basis of logarithmic image processing model with the inclusion of location parameters pertaining to on road weather degraded images. The proposed method utilizes multiple images to form a single image that is more visually pleasing. The parameters are chosen by performing enhancement with all practical values and then analyzing each output image using the proposed logarithmic region contrast measure and compared with other traditional measures.

This paper is organized as follows. Section 2 presents the necessary background information including the logarithmic image processing (LIP) model operator primitives and a review of contrast measures. Section 3 describes the proposed method for enhancement of weather degraded images and a new logarithmic region contrast measure, for obtaining the optimal parameters. Section 4 presents the computational complexity analysis, Section 5 presents the results of computer simulations for the enhancement of two sets of experimental data of weather degraded images. Section 6 presents concluding remarks.

\section{Background}

In this section the initial motivation of the LIP model and a review of contrast measures are included.

\subsection{Logarithmic Image Processing (LIP)}

In the LIP framework, the images are represented by mappings, called gray tone functions ( $f$ ) $[20,21]$ that are defined on the spatial domain and valued in the positive real number $\operatorname{set}[0, M)$, called the gray tone range. The transmittance of $f$ at point $(x, y)$ is given by:

$$
N_{f}(x, y)=\frac{\xi_{s}(x, y)}{\xi_{e}(x, y)}
$$

Where, $\xi_{\mathrm{s}}(\mathrm{x}, \mathrm{y})$ is the incoming flux,

$\xi_{\mathrm{e}}(\mathrm{x}, \mathrm{y})$ is the outgoing flux, 
In this perspective, the gray tone function $f$ is defined by:

$$
f(x, y)=M\left(1-N_{f}(x, y)\right)
$$

Eq. (1) and (2) illustrates the physical rationale and its consistency with the transmittance image formation model. The linear space constituted by the set of these gray tone functions ordered with a vector addition denoted $\oplus$ and a scalar multiplication, denoted $\otimes$ are defined in [20]. A mathematical meaning to the opposite and difference between two gray tone functions $f$ and $g$ is given [20] by:

$$
\begin{array}{r}
\Theta f=\frac{-M f}{M-f} \\
f \Theta g=M \frac{f-g}{M-g}
\end{array}
$$

\subsection{Contrast Measure}

A light that is observed by human eye may be expressed in differential form i.e. either as slightly brighter or much brighter than another light, but it is tough to allocate numerical values to such observations. It is however, expected to evaluate a contrast measure indirectly in terms of the minimum change in illumination which can be distinguished as a change in brightness.

If $L$ is the uniform background luminance observed by a human eye, and $\Delta L$ is the increase or decrease in that luminance, then the measure of the contrast defined [22] by:

$$
C_{w}=\frac{\Delta L}{L}
$$

is considered a measure of the perceptive power of the eye. This description of the relation between the discriminable threshold $\Delta L$ and the original luminance $L$ is known as the Weber-Fechner law.

For simple patterns, the Michelson contrast measure [23] is used to measure the contrast of a periodic pattern such as a sinusoidal grating and is defined as:

$$
C_{M}=\frac{L_{\max }-L_{\min }}{L_{\max }+L_{\min }}
$$

Eqs. (7) and (8) depicts the variability and deviation from a common ground. Fixed measurement of contrast using Eq. (8) is not appropriate because a pair of points of extreme brightness or darkness can determine the contrast of the whole image. For example, if a single over illuminated point or an especially under illuminated point is added to an average contrast image, the value of the Michelson contrast would increase significantly, but the apparent contrast may be reduced [22].

The definition of a local band limited contrast in the frequency domain defined in [22] provides a local contrast measure for every band that depends not only on the local energy at that band but also on the local background luminance as it varies from place to place in the image:

$$
C(x, y)=\frac{a(x, y)}{l(x, y)}
$$


Where, $a(x, y)$ - Filtered image

$$
l(x, y) \text { - Luminance mean image. }
$$

Eq. (7) is applicable to complex images and finds the contrast in the frequency domain.

Weber's law based contrast measure is defined in [26], for an image $I$, divided into $k_{1} \times k_{2}$ blocks with center $(k, l)$ of size $M_{1} \times M_{2}$ :

$$
E M E_{k_{1}, k_{2}}(\Phi)=\frac{1}{k_{1} k_{2}} \sum_{l=1}^{k 1} \sum_{k=1}^{k 2} 20 \ln \frac{I_{\mathrm{max} ; k, l}^{w}(\Phi, \text { par })}{I_{\mathrm{min} ; k, l}^{w}(\Phi, p a r)+c}
$$

where, $\Phi$ is a given transform, $I_{\max ; k, l}^{w}, I_{\min ; k, l}^{w}$ are the maximum and minimum luminance values in a block of the image, and $c$ is a small constant equal to 0.0001 to avoid dividing by 0 . This measure of enhancement is used to find the average ratio of maximum to minimum intensities in decibels. Another method of enhancement defined is based upon the concept of entropy [26]

$$
E M E E_{k_{1}, k_{2}}(\Phi)=\frac{1}{k_{1} k_{2}} \sum_{l=1}^{k 1} \sum_{k=1}^{k 2} \mu\left(\frac{I_{\max ; k, l}^{w}(\Phi, p a r)}{I_{\min ; k, l}^{w}(\Phi, p a r)+c}\right) \times \ln \frac{I_{\text {max } ; k, l}^{w}(\Phi, p a r)}{I_{\min ; k, l}^{w}(\Phi, p a r)+c}
$$

This is known as the measure of enhancement by entropy, or EME using entropy. The measures that are based on the Michelson contrast (Eq. (6)) are AME and AMEE defined in [27]. These gives the relationship between the spread and the sum of the two luminance values found in a small block.

$$
\begin{aligned}
& A M E=\left(\frac{1}{k_{1} k_{2}} \sum_{l=1}^{k_{1}} \sum_{k=1}^{k_{2}} 20 \ln \frac{I_{\mathrm{max} ; k, l}^{w}-I_{\mathrm{min} ; k, l}^{w}}{I_{\mathrm{max} ; k, l}^{w}+I_{\min ; k, l}^{w}+c}\right) \\
& A M E E=\frac{1}{k_{1} k_{2}} \sum_{l=1}^{k_{1}} \sum_{k=1}^{k_{2}} \mu\left[\frac{I_{\max ; k, l}^{w}-I_{\min ; k, l}^{w}}{I_{\max ; k, l}^{w}+I_{\min ; k, l}^{w}+c}\right]^{\mu} \times \ln \frac{I_{\max ; k, l}^{w}-I_{\min ; k, l}^{w}}{I_{\max ; k, l}^{w}+I_{\min ; k, l}^{w}+c}
\end{aligned}
$$

Next section describes the proposed method and a region based logarithmic contrast measure (LRCM) for finding the optimal parameters of the proposed method.

\section{Proposed Enhancement Method}

The following approach pertains at the outset to images obtained in the perspective of transmittance image formation LIP model:

Let $G$ be a set of weather degraded images:

$$
G=\left\{D_{1}, D_{2}, \ldots . D_{n}\right\}
$$

Where,

$D_{n}$. Weather degraded images/frame as captured from the silicon sensor based digital camera [25] at a time instant $t$ and location $l$ as depicted in Fig. 1. 


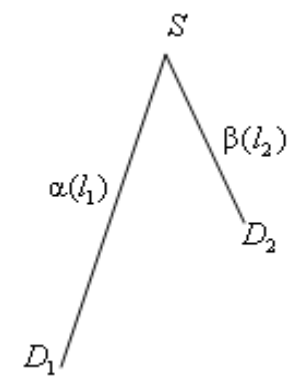

\section{Figure 1. Illustration of location parameters and capture points, of the degraded images $\left(D_{1}, D_{2}\right)$ with respect to a scene point $(S)$}

The sensitivity curve for human and silicon sensors as shown in Fig. 2 [24] evidently depicts a very low strength of silicon sensors sensitivity [25] in the human visual range as compared to human sensitivity, whereas beyond the range of human sensitivity, silicon sensitivity has significant strength.

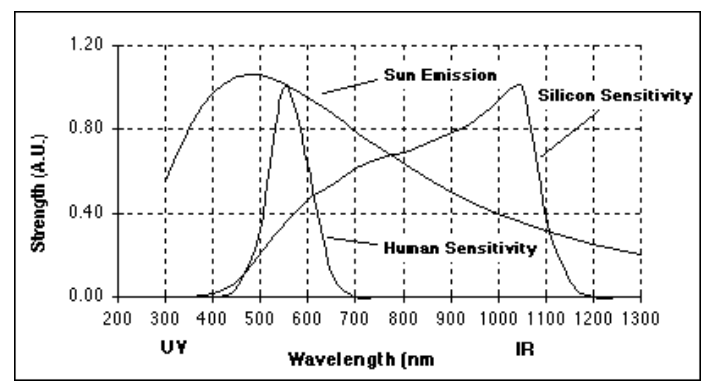

Figure 2. Sensitivity Curve

We attempt to define a spatial domain transformation function $(T)$ as a function of gray tones of degraded images and their respective location parameters to enhance and increase the information content of the weather degraded image captured from a silicon sensor based digital camera. This corresponds to the findings that the transmittance of a scene point gets modulated and it increases with increase in distance [6], [25]. $T$ is defined as:

$$
T=F\left\{\alpha\left(l_{1}\right), \beta\left(l_{2}\right), D_{n}\right\}
$$

For a set of two images and location parameters $\alpha\left(l_{1}\right)$ and $\beta\left(l_{2}\right)$ using both the conventional operators,$+ \times$ and LIP primitives $\oplus, \otimes, T_{s}$ and $T_{L}$ are defined as:

$$
\begin{gathered}
D_{s}=T_{s}\left\{\left(\alpha\left(l_{1}\right), \beta\left(l_{2}\right), D_{1}, D_{2}\right)\right\}=\alpha\left(l_{1}\right) \times D_{1}+\beta\left(l_{2}\right) \times D_{2}-\alpha\left(l_{1}\right) \beta\left(l_{2}\right) \times \frac{D_{1} D_{2}}{M} \\
D_{L}=T_{L}\left\{\left(\alpha\left(l_{1}\right), \beta\left(l_{2}\right), D_{1}, D_{2}\right)\right\}=\alpha\left(l_{1}\right) \otimes D_{1}+\beta\left(l_{2}\right) \otimes D_{2}-\alpha\left(l_{1}\right) \beta\left(l_{2}\right) \otimes \frac{D_{1} D_{2}}{M}
\end{gathered}
$$

Where, $D_{s}$ and $D_{L}$ are spatial domain transform images having distance information of the gray tone functions of both $D_{1}$ and $D_{2}$. 
The resultant enhanced images $E_{s, D 1}, E_{L, D 1}$ with respect to $D_{s}$ and $D_{L}$ is obtained by spatially transforming the degraded image $D_{1}$ (corresponding to location $l_{1}$ ) using:

$$
\begin{gathered}
E_{s, D 1}=T_{s}\left\{\left(D_{s}, D_{1}\right)\right\} \\
E_{L, D 1}=T_{L}\left\{\left(D_{L}, D_{1}\right)\right\}
\end{gathered}
$$

Figure 3 shows the block diagram of the proposed method.

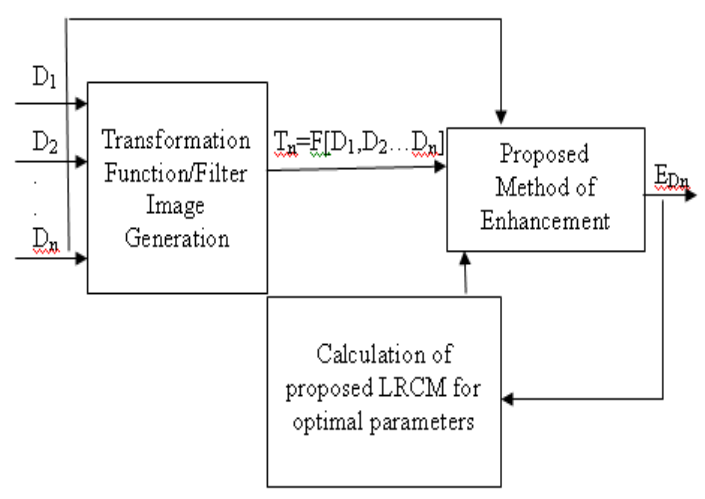

Figure 3. Block diagram of the proposed enhancement method

For finding the optimal location parameters $\alpha\left(l_{1}\right)$ and $\beta\left(l_{2}\right)$ a region based logarithmic contrast measure (LRCM) is presented. The defined measure is comprised of logarithmic subtraction and summation. It computes the contrast measure for a smallest discrete region (spatial domain) within the image, as compared to previously defined measures that calculate the same for each block of a divided image. The presented measure does not posses the disadvantage of range dependence while the contrast measures defined in Eqs. (8)-(11) are range dependent that change itself based on the maximum and minimum range [22].

$$
L R C M=20 \ln \left\{\sum_{m} \sum_{n}\left\{\begin{array}{c}
I(m, n) \Theta \\
\frac{1}{4} \oplus \sum_{i, j=0}^{1} I(m \pm i, n \pm j)
\end{array}\right)\right\}
$$

Next sections include an analysis of the computational complexity and results of computer simulation performed on two sets of experimental data of on road weather degraded images.

\section{Computational Complexity Analysis}

The evident implementation details of each conventional image enhancement method are currently unavailable. We provide a computational analysis on our proposed method. This calculates the spatial domain transformation function that computes one addition, one subtraction, two multiplications, one division and three LIP multiplications for each gray tone. Thus, the order of finding the transformation function is $O(n)$. Similarly the order of obtaining the enhanced image is also $O(n)$. For computing location parameters it uses one 
LIP subtractions and four LIP additions which results into a total of 6 subtractions, 4 additions, one multiplication, and one division, which must be calculated for every pixel of the resultant image. Thus, the complexity for the overall method for a set of two degraded images would be $O(3 n) \rightarrow O(n)$.

\section{Results}

In this section, we present the results of computer simulations of the proposed image enhancement method on two sets of experimental image data set of on road weather degraded images. We compare the results of the proposed method using the basic LIP model, method using conventional multiplicative parameters and the result of the method using logarithmic multiplicative parameters. We also compare the enhancement results of the proposed method with the result of histogram equalization.

For finding the optimum parameters; we use the presented measure LRCM and compare the same with those found using AMEE. Figure 4 and Figure 5 shows the results for two sets of experimental data of on road weather degraded images and their LRCM values. Table 1-2 compares the values of the contrast measures while increasing the location parameter $\alpha\left(l_{1}\right)$ for image data set of distant vehicle and letter board.

Table 1. Comparison of measures for increasing $\alpha\left(l_{1}\right)$ and $\beta\left(l_{2}\right)>1$ Distant vehicle image data set

\begin{tabular}{cccccc}
\hline \multicolumn{7}{c}{ Distant Vehicle } \\
\hline Alpha & AME & AMEE & EME & EMEE & LRCM \\
\hline 0.2 & 0.1057 & 0.1435 & 0.2089 & 10.5041 & 10.2748 \\
\hline 0.3 & 0.1064 & 0.1478 & 0.209 & 10.5491 & 10.8162 \\
\hline 0.4 & 0.1017 & 0.1562 & 0.2092 & 10.5691 & 10.8383 \\
\hline 0.5 & 0.1101 & 0.1718 & 0.2095 & 10.6038 & 10.7129 \\
\hline 0.6 & 0.1124 & 0.1886 & 0.2098 & 10.6463 & 10.8212 \\
\hline 0.7 & 0.1147 & 0.2069 & 0.2101 & 10.6996 & 11.0472 \\
\hline
\end{tabular}

It evidently indicates that measure value increases with increase in $\alpha\left(l_{1}\right)$ (corresponding to degraded image $D_{1}$ ). Tables 3-4 compares the values of the measures while decreasing the location parameter $\beta\left(l_{2}\right)$ (corresponding to degraded image $D_{2}$ ) for distant vehicle and letter board.

The values shown in Table 3 and 4 indicate a decrease in measure values with decrease in the value of location parameter $\beta\left(l_{2}\right)$. 
Table 2. Comparison of measures for increasing $\alpha\left(l_{1}\right)$ and $\beta\left(l_{2}\right)>1$ Letter board image data set

\begin{tabular}{cccccc}
\hline \multicolumn{7}{c}{ Letter Board } \\
\hline Alpha & AME & AMEE & EME & EMEE & LRCM \\
\hline 0.2 & 0.0946 & 0.0909 & 0.208 & 6.9267 & 10.7181 \\
\hline 0.3 & 0.0976 & 0.1032 & 0.2082 & 6.9795 & 10.7668 \\
\hline 0.4 & 0.1012 & 0.1196 & 0.2085 & 7.0484 & 10.8848 \\
\hline 0.5 & 0.1043 & 0.1359 & 0.2088 & 7.1165 & 11.0242 \\
\hline 0.6 & 0.1072 & 0.1529 & 0.2091 & 7.189 & 11.2057 \\
\hline 0.7 & 0.1097 & 0.1691 & 0.2094 & 7.2665 & 11.2055 \\
\hline
\end{tabular}

Table 3. Comparison of measures for decreasing $\beta\left(l_{2}\right)$ and $\alpha\left(l_{1}\right)<1$ Distant vehicle image data set

\begin{tabular}{cccccc}
\hline \multicolumn{5}{c}{ Distant Vehicle } \\
\hline Beta & AME & AMEE & EME & EMEE & LRCM \\
\hline 1.5 & 0.1147 & 0.2069 & 0.2101 & 10.7023 & 11.0472 \\
\hline 1.4 & 0.1133 & 0.1954 & 0.2099 & 10.6703 & 10.9047 \\
\hline 1.3 & 0.112 & 0.1855 & 0.2097 & 10.6388 & 10.8632 \\
\hline 1.2 & 0.1102 & 0.1724 & 0.2095 & 10.6125 & 10.8006 \\
\hline 1.1 & 0.1086 & 0.1617 & 0.2093 & 10.587 & 10.5732 \\
\hline 1 & 0.1071 & 0.1522 & 0.2091 & 10.5639 & 10.3789 \\
\hline
\end{tabular}

Figure 6(b) and 6(c) shows the results of enhancement of distant vehicle image data set with certain randomly chosen parameter values and with optimal parameter values respectively.

Table 4. Comparison of measures for decreasing $\beta\left(l_{2}\right)$ and $\alpha\left(l_{1}\right)<1$ Letter board image data set

\begin{tabular}{cccccc}
\hline \multicolumn{6}{c}{ Letter Board } \\
\hline Beta & AME & AMEE & EME & EMEE & LRCM \\
\hline 1.5 & 0.1097 & 0.1691 & 0.2094 & 7.2665 & 11.2055 \\
\hline 1.4 & 0.1091 & 0.1652 & 0.2093 & 7.2458 & 11.2553 \\
\hline 1.3 & 0.1082 & 0.159 & 0.2092 & 7.2158 & 11.2425 \\
\hline 1.2 & 0.1072 & 0.1526 & 0.2091 & 7.1875 & 11.0915 \\
\hline 1.1 & 0.1057 & 0.1439 & 0.2089 & 7.15 & 10.9561 \\
\hline 1 & 0.1049 & 0.1392 & 0.2088 & 7.1303 & 10.8452 \\
\hline
\end{tabular}

It is clearly evident that the image enhanced with the optimal parameters is visually better than the other. Figure 7(b) and Figure 7(c) show the results of letter board data set with similar outcome. Figure 6(d), Figure 7(d) shows the plot between LRCM and parameters for 
the purpose of choosing an optimal value as their local maxima. The optimal values are then compared with the values obtained from the plot between AMEE and parameters; Figure 6(e), Figure 7(f). Figure 7(e) shows the result of the proposed enhancement method with the parameters found as the local maxima of Figure 7(f). It is thus illustrated that we obtained similar optimal values for the distant vehicle data set when using both LRCM as well as AMEE measure, while the values found from both the measures differ for the letter board data set.

The original degraded images in Figure 4(a), (e) and Figure 5(a), (e) are images that are captured by a conventional silicon sensor camera (Sony DSC-W210).

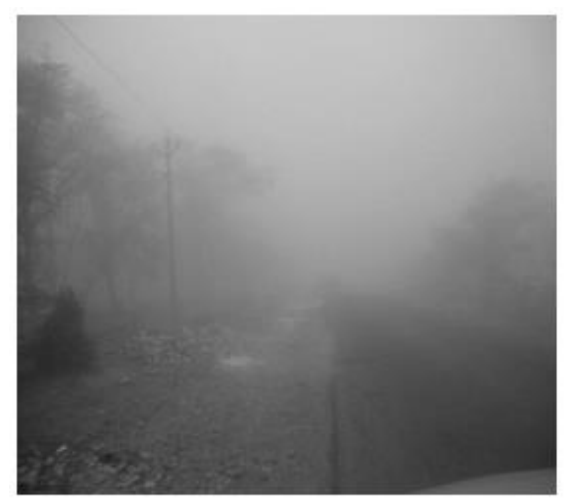

(a)

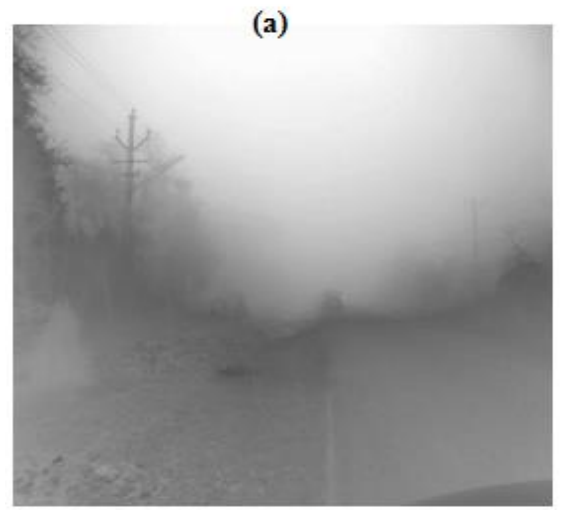

(c)

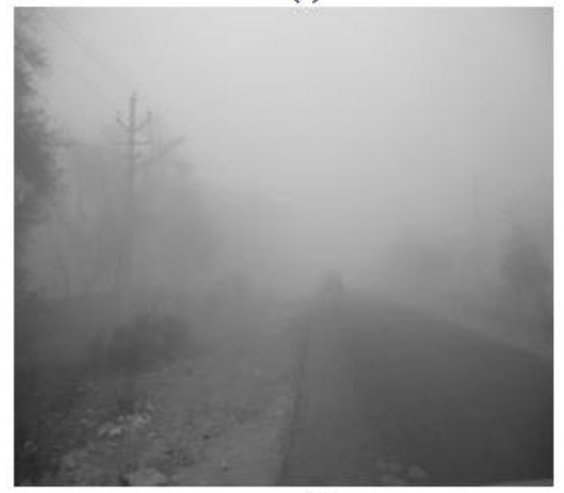

(e)

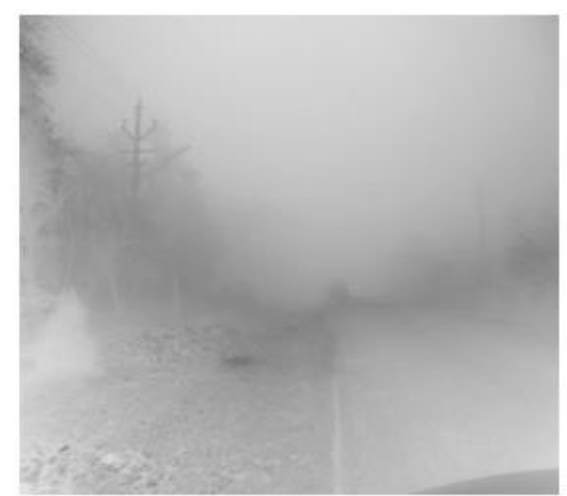

(b)

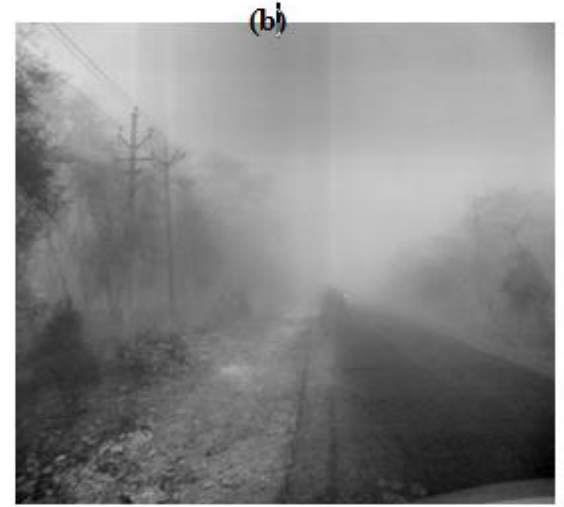

(d)

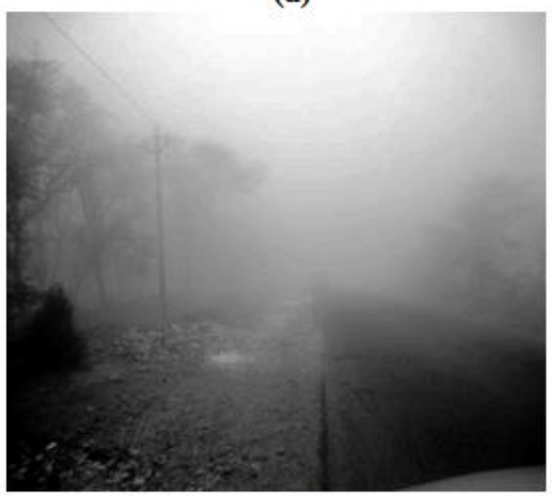

(f)

Figure 4. (a) Degraded Image D1 (LRCM=10.6295) (b) Result of method using basic LIP model (LRCM=9.6831) (c) Result of method using conventional multiplicative parameters $(\mathrm{LRCM}=10.9504)$ (d) Result of method using optimal 
logarithmic multiplicative parameters (LRCM=11.0153) (e) Degraded Image D2 (LRCM=10.6536) (f) Result of Histogram Equalization (LRCM=11.0074)

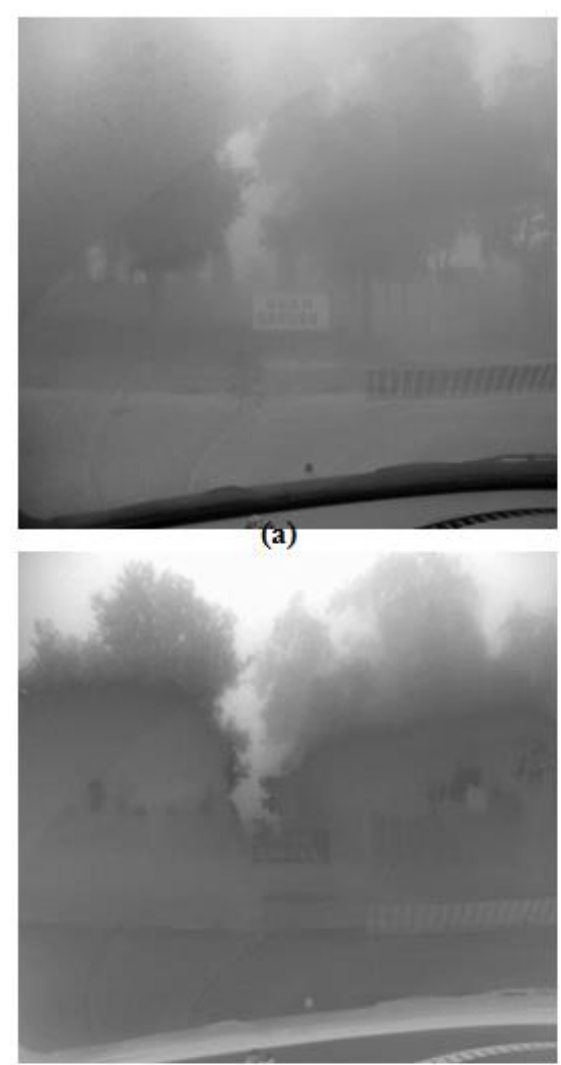

(c)

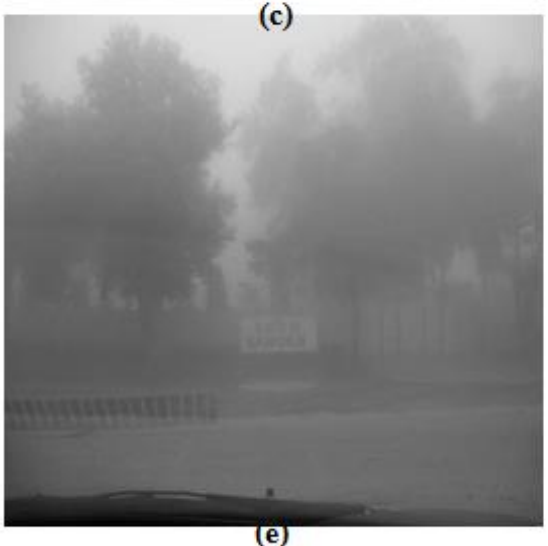

(e)

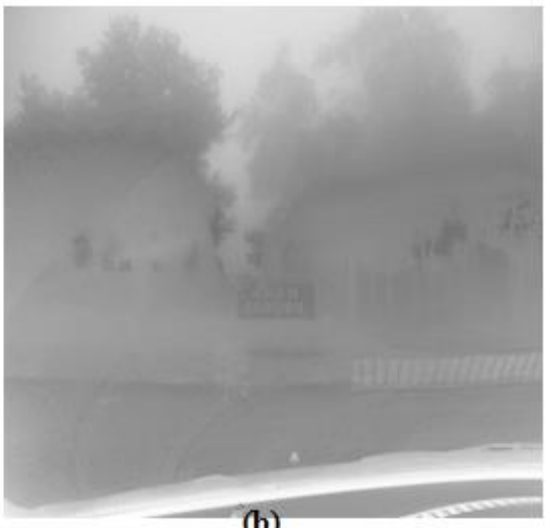

(b)

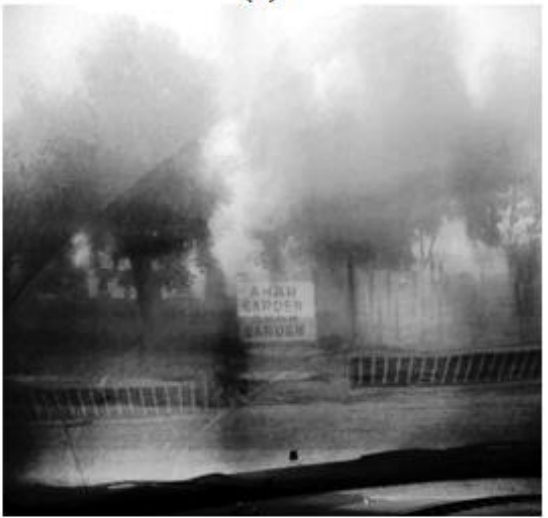

(d)

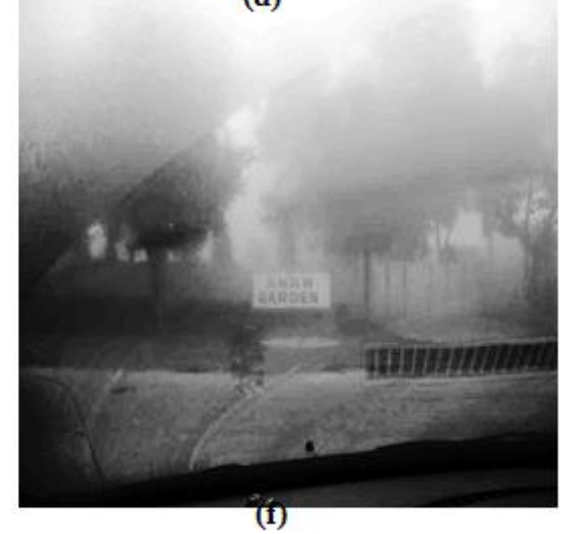

Figure 5. (a) Degraded Image D1 (LRCM=10.9512) (b) Result of method using basic LIP model (LRCM=10.8415) (c) Result of method using conventional multiplicative enhancement parameters (LRCM=11.4146) (d) Result of method using optimal logarithmic multiplicative location enhancement parameters

(LRCM=11.5099) (e) Degraded Image D2 (LRCM=11.0056) (f) Result of Histogram Equalization (LRCM=11.5052) 


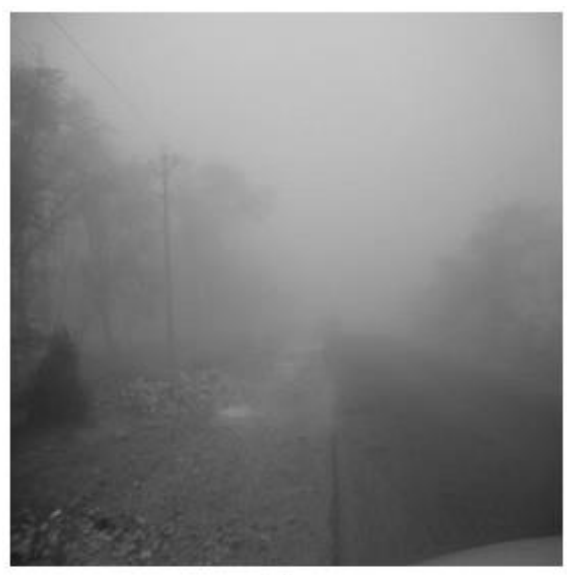

(a)

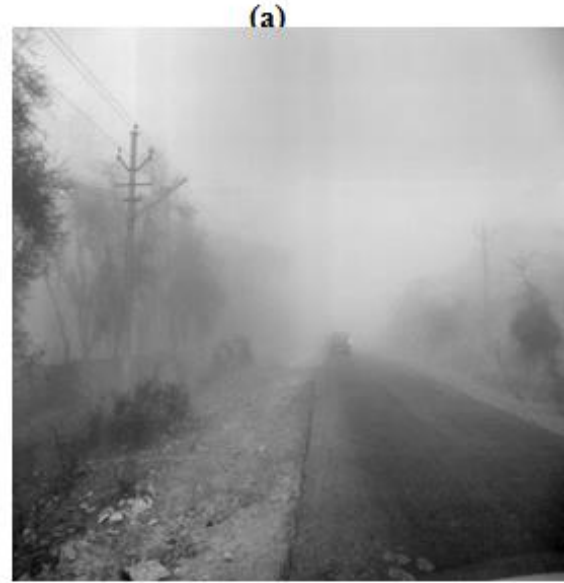

(c)

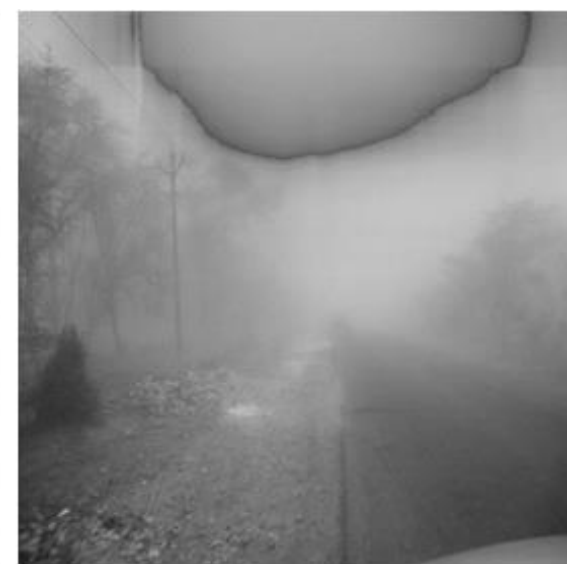

(b)

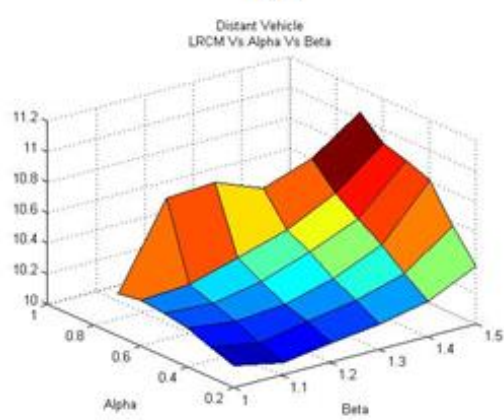

(d)

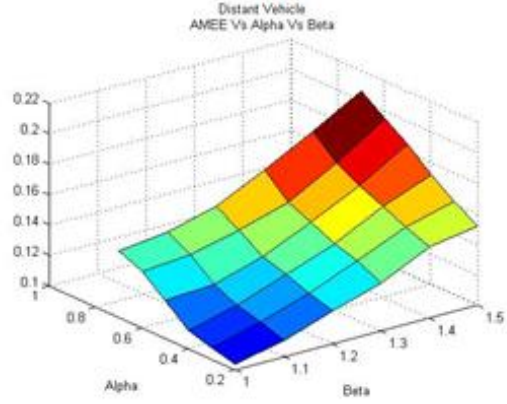

(e)

Figure 6. (a) Degraded Image D1 (LRCM=10.6295) (b) Result of enhancement with logarithmic location enhancement parameter ${ }_{\alpha\left(l_{i}, \theta_{1}\right)}=0.2, \beta\left(l_{2}, \theta_{2}\right)=1.5$

( $L R C M=10.2748)$ (c) Result of enhancement with optimal logarithmic location enhancement parameter ${ }_{\alpha\left(l_{1}, \theta_{1}\right)}=0.7, \beta\left(l_{2}, \theta_{2}\right)=1.5$ (LRCM=11.0153) chosen from local maxima of LRCM Vs Alpha Vs Beta (d) LRCM Vs Alpha Vs Beta (e) AMEE Vs Alpha Vs Beta 


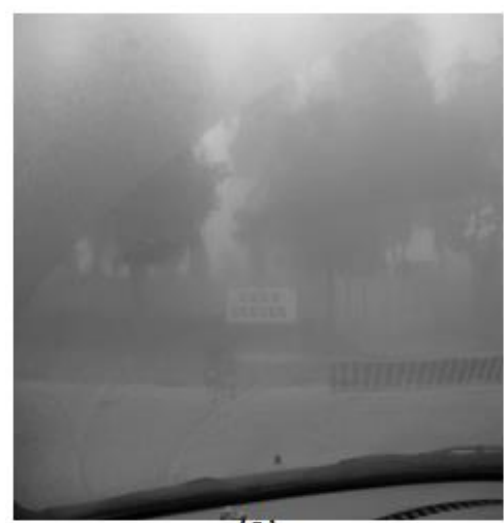

(a)

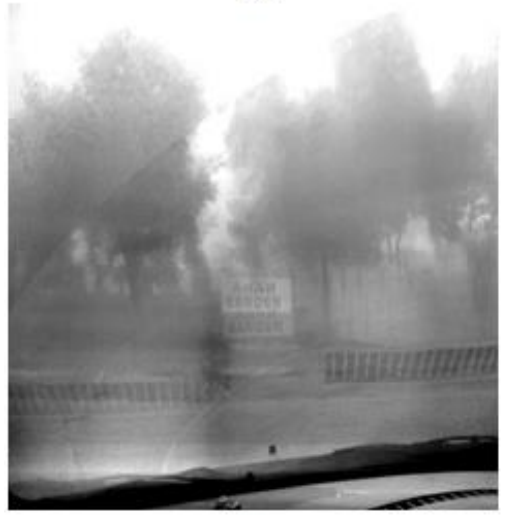

(c)

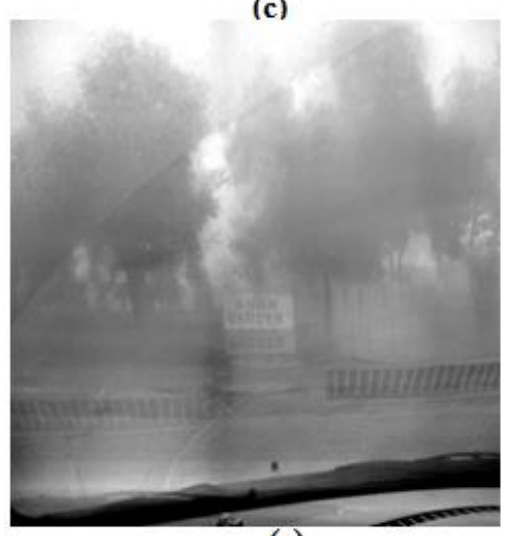

(e)

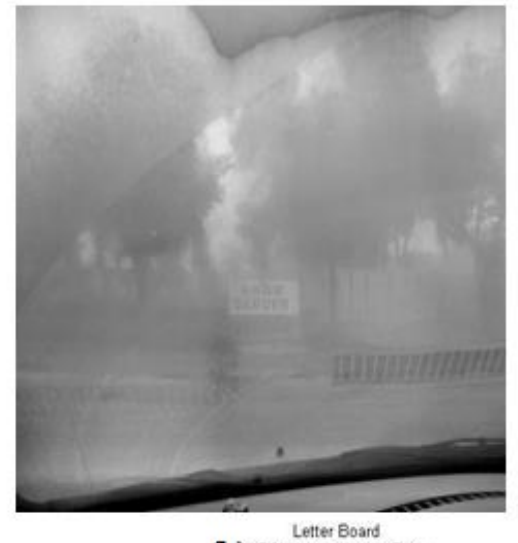

(b) LRCM Vetter Alpoard ${ }^{8}$ Beta

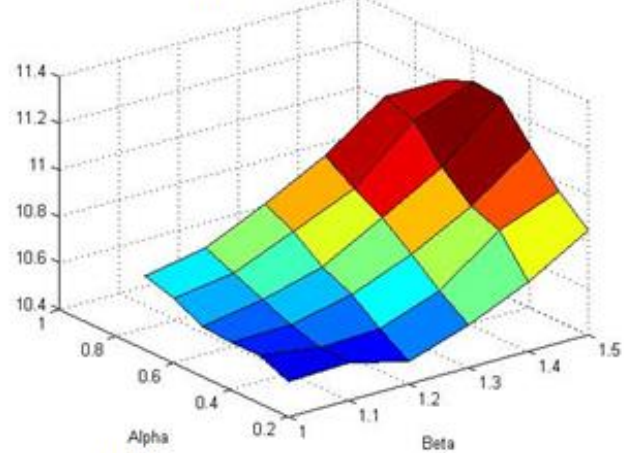

(d)

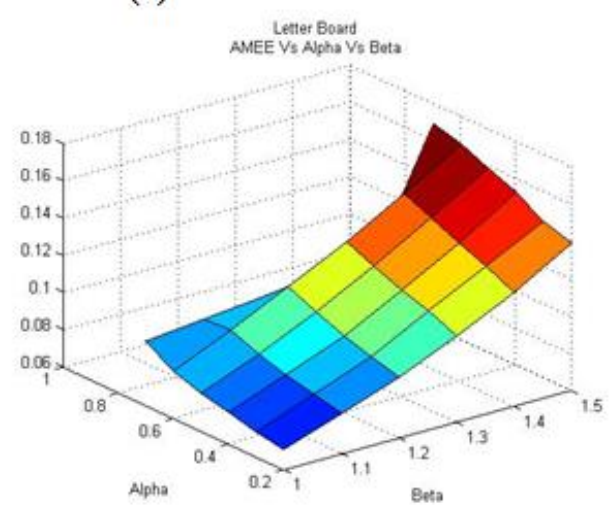

(f)

Figure 7. (a) Degraded Image D1 (LRCM=10.9512) (b) Result of enhancement with logarithmic location enhancement parameter $\alpha\left(l_{l}\right)=0.3, \beta\left(l_{l}\right)=1.5$

(LRCM=10.7668) (c) Result of enhancement with optimal logarithmic location enhancement parameter ${ }_{\alpha\left(l_{l}\right)}=0.6, \beta\left(l_{i}\right)=1.4(\mathrm{LRCM}=11.2553)$ chosen from local maxima of LRCM Vs Alpha Vs Beta (d) LRCM Vs Alpha Vs Beta (e) Result of enhancement with location enhancement parameter ${ }_{\alpha(l)}=0.7, \beta\left(l_{l}\right)=1.5$ (=11.2055) chosen from local maxima of AMEE Vs Alpha Vs Beta (f) AMEE Vs Alpha Vs Beta 


\section{Conclusion}

In this paper, a new weighted LIP model based image enhancement method was presented. Two location based parameters with LIP multiplication are used in the method which produces results that are better than the method without parameters; the method using parameters with conventional multiplication and global histogram equalization. This was illustrated both by observing the enhanced results as well as their contrast measure values. The method that used location based parameters with LIP multiplication provided the maximum value of the contrast measure and hence increased information. A novel region based contrast measure called LRCM was presented and compared with other traditional measures. The presented measure was used for finding the optimum parameters for the proposed method. It was found that the presented measure commensurate with a conventional measure in one case while it outperforms for the other out of the experiments carried out on two data sets.

\section{References}

[1] M. Jourlin and J. C. Pinoli, "Logarithmic image processing: The mathematical and physical framework for the representation and processing of transmitted images", Adv. Imag. Electron Phys., vol. 115, (2001), pp. 129-196.

[2] I. E. Gordan, “Theories of Visual Perception”, Wiley, New York, (1989).

[3] R. C. Gonzalez and P. Wintz, "Digital Image Processing", Addison-Wesley, (1987).

[4] A. K. Jain, "Fundamentals of Digital Image Processing", Prentice Hall, (1989).

[5] H. Shvayster and S. Peleg, "Pictures as elements in a vector space", Proc. IEEE Conf. Comput. Vision Pattern Recogn, Washington, (1983) June, pp. 442-446.

[6] S. G. Narasimhan and S. K. Nayar, "Vision and the Atmosphere", Int'l J. Computer Vision, vol. 48, (2002), pp. 233-254.

[7] S. G. Narasimhan and S. K. Nayar, "Interactive Deweathering of an Image Using Physical Models", Proc. IEEE Workshop Color \& Photometric Methods in Computer Vision, (2003).

[8] Y. Y. Schechner, S. G. Narasimhan and S. K. Nayar, "Polarization-Based Vision through Haze", Applied Optics, vol. 42, (2003), pp. 511-525.

[9] S. G. Narasimhan, C. Wang and S. K. Nayar, "All the Images of an Outdoor Scene", Proc. European Conf. Computer Vision, (2002), pp. 148-162.

[10] J. P. Oakley and B. L. Satherley, "Improving Image Quality in Poor Visibility Conditions Using a Physical Model for Contrast Degradation”, IEEE Trans. Image Processing, vol. 7, (1998), pp. 167-179.

[11] Y. Yitzhaky, I. Mor, A. Lantzman and N. S. Kopeika, "Direct method for restoration of motion-blurred images", J. Opt. Soc. Am., vol. 15, no. 6, (1998) June.

[12] R. T. Tan, N. Petersson and L. Petersson, "Visibility enhancement for roads with foggy or hazy scenes", Proc. IEEE Intelligent Vehicles Symposium, (2007) June.

[13] J. Dusek and K. Roubik, "Testing of new models of the human visual system for image quality evaluation", Proc. IEEE 7th Int. Symp. Signal Process. Appl., vol. 2, (2003) July, pp. 621-622.

[14] K. K. Sharma, et al., "Contrast Enhancement Algorithm for fog-degraded images", Proceedings of Third National Conference on Vision, Pattern Recognition, Image Processing and Graphics,(2010) November, pp. 52.

[15] M. K. Kundu and S. K. Pal, “Thresholding for edge detection using human psychovisual phenomena”, Pattern Recognit. Lett. vol. 4, no. 6, (1986) December, pp. 433-441.

[16] R. A. Nobakht and S. A. Rajala, "An image coding technique using a human visual system model and image analysis criteria”, Proc. IEEE Int. Conf. Acoust., Speech, Signal Process., vol. 12, (1987) April, pp. 13581361.

[17] G. Buchsbaum, “An analytical derivation of visual nonlinearity”, IEEE Trans. Biomed. Eng., vol. BME-27, no. 5, (1980) May, pp. 237-242.

[18] C. Munteanu and A. Rosa, "Gray-scale image enhancement as an automatic process driven by evolution", IEEE Trans. Syst., Man, Cybern., vol. 34, no. 2, (2004) April, pp. 1292-1298.

[19] D. J. Granrath, "The role of human visual models in image processing", Proc. IEEE, vol. 69, (1981) May, pp. 552-561. 
[20] M. Jourlin and J. C. Pinoli, “A Model for logarithmic image processing”, J. Microsc., vol. 149, (1988) January, pp. 21-35.

[21] J. C. Pinoli, "The logarithmic image processing model: Connections with human brightness perception and contrast estimators", Journal of Mathematical Imaging and Vision, vol. 7, (1997), pp. 341-358.

[22] E. Peli, "Contrast in complex images", J. Opt. Soc. Amer. A, vol. 7, (1990), pp. 2032-2040.

[23] A. A. Michelson, "Studies in Optics", Chicago, IL: Univ. Chicago Press, (1927).

[24] The camera technical, http://www.maxmax.com/camera_technical.

[25] B. S. Carlson, "Comparison of Modem CCD and CMOS Image Sensor Technologies and Systems for Low Resolution Imaging”, IEEE Sensors Conference, (2002).

[26] S. S. Agaian, K. Panetta and A. M. Grigoryan, "A new measure of image enhancement", presented at the IASTED Int. Conf. Signal Processing Communication, Marbella, Spain, (2000) September 19-22.

[27] S. Agaian, B. Silver and K. Panetta, "Transform coefficient histogram based image enhancement algorithms using contrast entropy”, IEEE Transactions on Image Processing, vol. 16, no. 3, (2007) March, pp. 741-758.

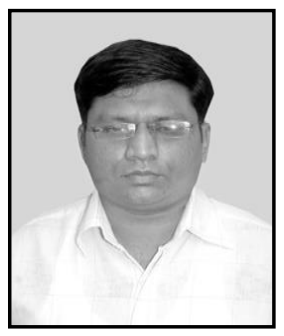

Hitendra Gupta was born in India, in June 1979. He received his B.E. in Electronics Engineering in 2001, and M.Tech (Gold Medal) from Malaviya National Institute of Technology, Jaipur, India in 2008. He is presently working with Jaipur Engineering College and Research Centre, Jaipur, India. His research interest includes image processing algorithms, signal processing systems.

E-mail: hitendra_gupta@ rediffmail.com

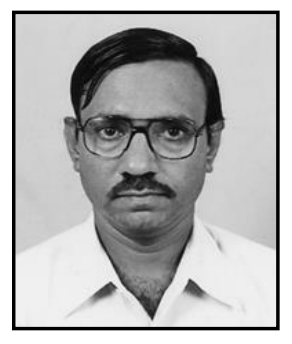

Kamalesh Kumar Sharma received the B.E. (honors), and M.E. (honors) degrees in electronics and communication engineering from Malaviya National Institute of Technology, Jaipur, India, in 1990 and 2001, respectively. Presently, he is working as an Associate Professor with the Malaviya National Institute of Technology. From 1990 to 1997, he served as a Lecturer with the Electronics and Communication Engineering Department, Malaviya National Institute of Technology. He joined the Indian Telecommunication Service for a year in 1997 as an Assistant Divisional Engineer Telecom. From 1998 to 2003, he again served as a Lecturer with Malaviya National Institute of Technology. He was deputed to the Indian Institute of Technology, New Delhi, India, from 2003 to 2006, for pursuing the Ph.D. degree under the quality improvement program of the Government of India. His research interests include sampling theory of signals, image processing, and fractional transforms. He is a life member of I.E.T.E., India.

E-mail: kksharma_mrec@yahoo.com 


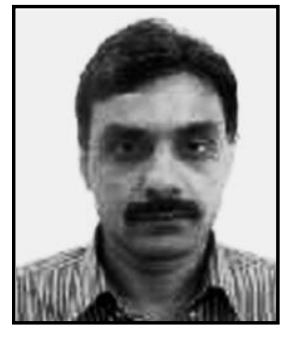

Shiv Dutt Joshi received the B.E. (honors) in electrical and electronics engineering from Birla Institute of Technology, Pilani, India, in 1981, the M.Tech. degree in communications and radar engineering, and the Ph.D. degree, both from the Indian Institute of Technology, Delhi, India, in 1983 and 1988, respectively. He worked as a Lecturer with the Delhi Institute of Technology for a year from 1988 to 1989 and joined the Indian Institute of Technology as a faculty member in May 1989, where he is currently a Professor with the Electrical Engineering Department. His research interests include development of fast algorithms for stochastic signal processing, speech processing, and modeling of stochastic processes. Dr. Joshi was a recipient of the AES Award from the IEEE AES/COM Society, Indian Chapter, for the year 1986.

E-mail: sdjoshi@ee.iitd.ac.in 
International Journal of Signal Processing, Image Processing and Pattern Recognition Vol.6, No.6 (2013)

itd.ac.in 